\title{
A Power-Optimized Handover strategy in Hybrid Satellite-Terrestrial Networks
}

\author{
Huiping $X u^{1, a^{*}}$, Jinchun Gao ${ }^{2, b}$, Hefei Hu ${ }^{3, c}$, Dongming Yuan ${ }^{4, d}$, Jing Ran ${ }^{5, e}$ \\ ${ }^{1}$ Dept. of Electronic Engineering, Beijing Univ. of Post and Telecommunications, Beijing, 100876, \\ China \\ abupt_xuhp@163.com
}

Keywords: Satellites Power-optimized Markov decision process (SPO-MDP); Handover Strategy; Hybrid Satellite-Terrestrial Networks (HSTNs)

\begin{abstract}
Satellite communications provide large-scale coverage, so they can be integrated with terrestrial networks to implement a global wireless system, namely HSTNs. However, the network resources of satellite systems, such as the bandwidth and power, are extremely limited. This paper proposes a handoff strategy to minimize the power consumption of satellite systems required in serving a traffic flow in a hybrid Satellite-Terrestrial system, while guaranteeing the user QoS. It first established a speed-sensing based handoff model integrated with both horizontal and vertical handoff. To further minimize the power consumption, an improved Markov decision algorithm for vertical handover, namely SPO-MDP, is brought up. Simulation results show that the proposed model and scheme can lead to a lower power consumption and fewer handoff times in satellite systems compared to several existing schemes and meanwhile keeps the call drop rate at a respectively low level.
\end{abstract}

\section{Introduction}

The HSTNs consist of two parts -Mobile Satellite Systems (MSS) and terrestrial network systems. Terrestrial systems include heterogeneous networks such as cellular network, WLAN, WiMAX [1]. MSS has the advantages of wider coverage and disaster tolerance because satellite spot beams cover almost the whole surface of the planet. However, Satellite network capacity is limited by the relatively small bandwidth assigned to satellite communication and the high power required for transmission [2]. Furthermore, the cost of satellite communication construction is much higher than the terrestrial ones. To provide mobile terminals in HSTNs with constant service, it's unavoidable to carry out handovers during the mobility of users. However, the power consumption in satellites during a traffic flow can be minimized. This paper focuses to address the problem of power optimization in satellite systems during the handover process of a Mobile Node (MN) roaming in the HSTNs.

\section{Previous research}

In existing literature, there exist numerous handover strategies for terrestrial heterogeneous wireless networks [3]. According to the parameters used for vertical handover decision (VHD), these strategies can be divided into categories of: Received Signal Strength (RSS) based ones, network connection time based ones, power consumption based ones etc. In the work of Kantubukta[4], an energy and quality of service aware and technique for order preference by similarity to ideal solution (TOPSIS) based vertical handover strategy was proposed. In [5], the method of multiple attribute decision making (MADM) was used in the vertical handover scheme. In general, satellite network is also one of the heterogeneous wireless networks, so the existing VHD strategies can be used in HSTNs. However, the network characters and handover scenario in HSTNs are different with those in terrestrial networks. The VHD strategies mentioned above need to be improved to suit for the HSTNs. The power-optimized hybrid overall handover model and SPO-MDP handover scheme proposed in this paper is based on the work of Yujae Song in [6] but modified to match the unique situations in HSTNs. 


\section{The proposed hybrid handover model and the SPO-MDP scheme}

HSTNs model. In this paper, we mainly discuss the HSTNs formed by GEO satellites for GEO satellites maintain a respectively static topology, thus the mobility of satellites can be ignored in the HSTNs model. Each of the satellites spot beam covers a large area containing hundreds of small terrestrial base stations which are distributed randomly and intersectional.

MN model. The user (i.e. MN) traverses through the overlapped areas of satellite and terrestrial networks while changing velocity from time to time. Let each active $\mathrm{MN}$ be represented by a traffic flow that consists of $\mathrm{L}$ frames. After transmitting the $\mathrm{L}$ frames, an active $\mathrm{MN}$ becomes inactive and waits to start another conversation. At each time point, the $\mathrm{MN}$ is connected to only one network and suppose that during a single frame, both the terminal velocity and the network states stay stable. The overall architecture of the HSTNs can be represented as Fig.2.Usually, the velocity of a MN can be ranging from 0 to $1000 \mathrm{~km} / \mathrm{h}$. Some typical mobility situations could be: airplane (about $800 \mathrm{~km} / \mathrm{h}$ ), high-speed railway (about $200 \mathrm{~km} / \mathrm{h}$ ), common vehicles $(60 \mathrm{~km} / \mathrm{h}$ ) and walking (below $5 \mathrm{~km} / \mathrm{h}$ ).In order to model the user mobility, the velocity can be divided into two level -high speed and low speed using the Fuzzy Theory. In the later handover strategy, we established two different handover modes for the $\mathrm{MN}$ at high speed and low speed.

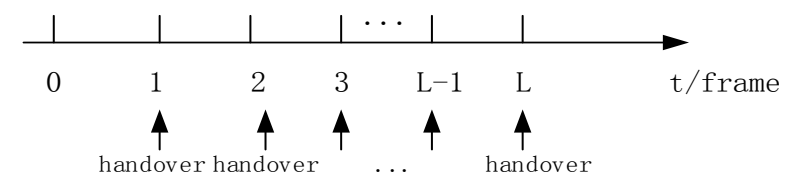

Fig.1 Handover timing in a traffic flow (L frames)

The hybrid handover model for HSTNs. In this section, a speed-sensing based hybrid handover

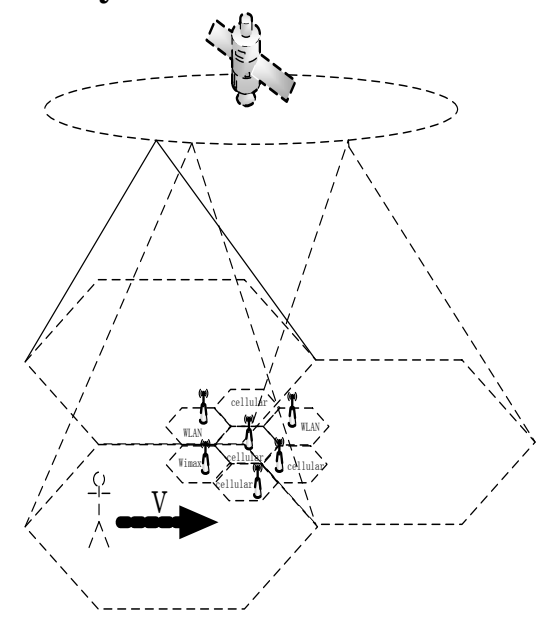
model involved with both horizontal and vertical handover is proposed. According to Figure.1, there are L frames in a traffic flow, the handover process happens at the end of each frame except the 0 frame, so there are $\mathrm{L}$ handover epochs during one conversation. In order to minimize the power consumption of the satellite networks, the MN shall make a full use of terrestrial networks and should not handoff to satellite networks if terrestrial networks can meet the QoS requirements of users. Based on this, a handover mode choosing module is set in the algorithm. Specifically, when the user speed is sensed as low, it goes directly to scan the available networks around the node and takes a horizontal handover in terrestrial networks at most of the time. In this case, the power consumption of satellites are saved effectively.

Fig. 2 Architecture of HSTNs

When the user speed is sensed as a high speed, it goes into the high speed handover mode. Since in this model, the $\mathrm{MN}$ is moving very fast so the networks an $\mathrm{MN}$ experience during a traffic flow are changing dramatically frequent, thus the MN has to take frequent handovers which could lead to high drop rate and miserable QoS due to high delay, especially when $\mathrm{MN}$ traverses through some networks which provide extremely small radius of service. While the satellite networks have a large radius of service and can help to maintain a continuous service. In this case, MN should take a vertical handover process to decide whether to connect to the satellite networks or terrestrial networks. In the work of Yujae Song and Peng-Yong, a Markov Decision Process (MDP) based vertical handover scheme was proposed. However, the MDP scheme needs modifications to adapt to the situation of HSTNs in this paper. First, the MDP scheme aims to optimize the overall power consumption of all the networks in the system. While in the HSTNs, it's more appropriate to minimize the power consumed in satellite networks because they are so limited. Second, in the MDP scheme, user QoS was not guaranteed. So the SPO-MDP scheme proposed in this paper adds a user QoS function to the cost function of the decision process. 
SPO-MDP scheme. In this section, we will describe the SPO-MDP scheme in detail. A MDP is defined as a tuple $\mathrm{T}=$ $(\mathrm{S}, \mathrm{A}, \mathrm{T}, \mathrm{r})$, where $\mathrm{S}$ is state space, $\mathrm{A}=$ $\{1,2\}$ is the set of all possible solutions, $\mathrm{T}\left[\mathrm{s}^{\prime} \mid \mathrm{s}, \mathrm{a}\right]$ is the transition probability from state $\mathrm{s}=$ $\left[\mathrm{m}, q_{1}, q_{2}\right] \in \mathrm{S}$ to $\mathrm{s}=\left[\mathrm{m}^{\prime}, q_{1}^{\prime}, q_{2}^{\prime}\right] \in \mathrm{S}^{\prime}$ after taking action $a \in A$ in state $s$, and $\mathrm{c}(\mathrm{s}, \mathrm{a})$ is the cost function of performing action $\mathrm{a}$ in state $\mathrm{s}$ during a frame. Let $M$ be the set of available networks for a $\mathrm{MN}$ such that $\mathrm{M}=$ $\{1,2\}$, where 1 stands for the satellite networks and 2 stands for terrestrial networks. We define link $\mathrm{n}$ as a wireless channel that connects the $\mathrm{MN}$ to network $m \in \mathrm{M}$. Let $Q^{m}(m=$ 1,2 ) stands for the quality of the wireless link $\mathrm{m}$. Therefore, the state space is $=\{1,2\} \times Q^{1} \times Q^{2}$.

According to the finite-Markov channel ( FSMC) model in [7], the time varying link quality can be quantized into I discrete values and stays stable during the service time of a frame. let $q_{m}$ represents the channel quality of link $\mathrm{m}$ and $0=R_{0}<R_{1}<\cdots<$ $R_{I-1}<R_{I}=\infty$ represents the predefined received signal strength (RSS) thresholds. Then $q_{m}$ is said to have

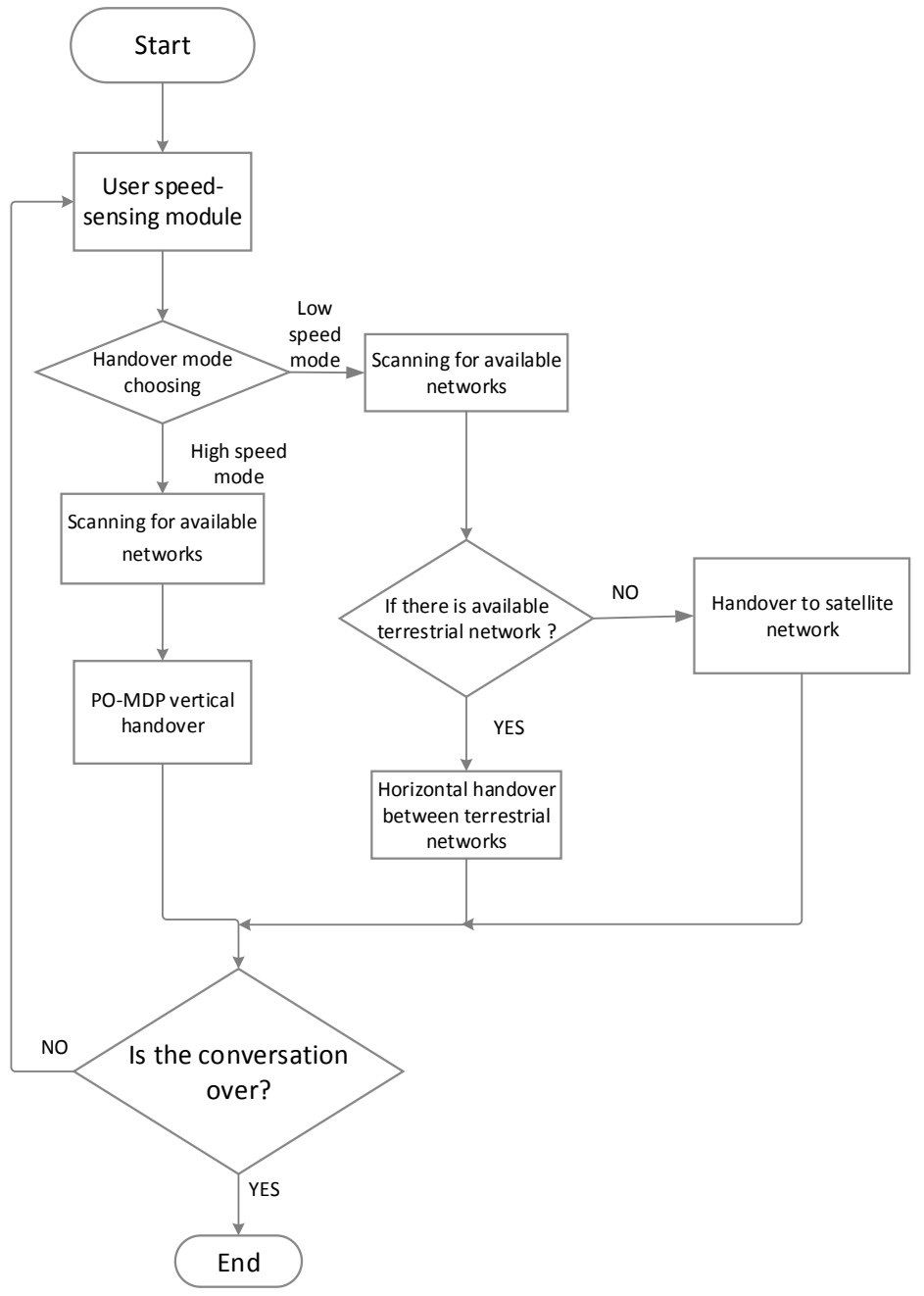

Fig. 3 Flow chart of the overall handover strategy the quality level of $R_{i}$, if $R_{i} \leq r_{m}(t)<R_{i+1}$, where $r_{m}(t)$ is the real-time RSS of link m. Denote $\bar{r}$ as the mean RSS, and $f_{R}(r)=\frac{1}{\bar{r}} \exp \left\{-\frac{r}{\bar{r}}\right\}$ is the probability density function of $\mathrm{R}$ which is exponential.

The probability $p_{i}=P\left[q_{m}=R_{i}\right]$ is state-steady and can be calculated by $p_{i}=P\left[q_{m}=R_{i}\right]=$ $\int_{R_{i}}^{R_{i+1}} f_{R}(r) d r=\exp \left\{-\frac{R_{i}}{\bar{r}}\right\}-\exp \left\{-\frac{R_{i+1}}{\bar{r}}\right\}$. The transition probability from a link quality to another can be represented by

$$
\begin{aligned}
& p_{i, i+1}=\frac{D_{i+1}}{s_{m} p_{i}}, i=0,1, \ldots, I-2 \\
& p_{i, i-1}=\frac{D_{i}}{s_{m} p_{i}}, i=1,2, \ldots, I-1 \\
& p_{0,0}=1-p_{0,1} \\
& p_{I-1, I-1}=1-p_{I-1, I-2} \\
& p_{i, i}=1-p_{i, i-1}-p_{i, i+1, i=1,2, \ldots, I-2}
\end{aligned}
$$

Where $D_{i}=\sqrt{\frac{2 \pi R_{i}}{\bar{r}}} f_{D} \exp \left\{-\frac{R_{i}}{\bar{r}}\right\}$ is the expected number of downward crossings in RSS, when maximum Doppler frequency is $f_{D}$, and $s_{m}$ is the service rate of network $m$. Thus,

$$
\mathrm{T}\left[\mathrm{s}^{\prime} \mid \mathrm{s}, \mathrm{a}\right]=\left\{\begin{array}{c}
P\left[q_{1}^{\prime} \mid q_{1}\right] * P\left[q_{2}^{\prime} \mid q_{2}\right], m^{\prime}=a \\
0, \text { otherwise }
\end{array}\right.
$$

The cost function $\mathrm{c}(\mathrm{s}, \mathrm{a})$ is defined as the division of the power cost function $\mathrm{p}(\mathrm{s}, \mathrm{a})$ and user QoS reward function $\mathrm{u}(\mathrm{s}, \mathrm{a})$. That is:

$$
\mathrm{c}(\mathrm{s}, \mathrm{a})=\mathrm{p}(\mathrm{s}, \mathrm{a}) / u(s, a)
$$


$\mathrm{u}(\mathrm{s}, \mathrm{a})$ is defined as follows:

$$
\mathrm{u}(\mathrm{s}, \mathrm{a})=\left\{\begin{array}{c}
1, q_{a} \geq H_{r s s} \\
\left(q_{a}-L_{r s s}\right) /\left(H_{r s s}-L_{r s s}\right), L_{r s s} \leq q_{a} \leq H_{r s s} \\
0, q_{a} \leq L_{r s s}
\end{array}\right.
$$

where $H_{r S s}$ and $L_{r S S}$ represent the highest and lowest RSS the connection requires.

The cost $\mathrm{p}(\mathrm{s}$, a) stands for power consumption of chosen network a to transmit one frame to an $\mathrm{MN}$ and is defined as the sum of transmission power from network $\mathrm{a}-P_{a, t x}$ and the circuit power of network a,and also the handover cost $\mathrm{h}(\mathrm{s}$, a) as given below:

$$
\mathrm{p}(\mathrm{s}, \mathrm{a})= \begin{cases}w *\left(\frac{b_{\text {frame }}}{s_{n}}\left(P_{a, t x}+P_{a, c}\right)+h(s, a)\right), & a=1 \\ \frac{b_{\text {frame }}}{s_{n}}\left(P_{a, t x}+P_{a, c}\right)+h(s, a), & a=2\end{cases}
$$

where $\mathrm{w}$ is the weight factor that denotes the ratio of bias for satellite networks when considering the power consumption, $b_{\text {frame }}$ is the number of frames in a traffic flow.

With the power control, $P_{a, t x}$ depends on link quality and can be formulated as follows:

$$
P_{a, t x}=\left\{\begin{array}{c}
\frac{\left(p_{a, \min }-p_{a, \max }\right)\left(\left(r-r_{0}\right)\right)}{\left(r_{t h}-r_{0}\right)}+p_{a, \max }, r_{0} \leq r \leq r_{t h} \\
p_{a, \min }, \quad r_{t h} \leq r \leq r_{I-1}
\end{array}\right.
$$

Where $p_{a, \max }, p_{a, \min }$ denotes maximum and minimum transmission power supported by the chosen network a.

The handover cost function $\mathrm{h}(\mathrm{s}$, a) reflects the power consumption of networks incurred by signaling exchanges and processing loads during the vertical handover, as given below (let $h_{i, a}$ denotes the power consumption required for handover from $i$ to $a$ ):

$$
\mathrm{h}(\mathrm{s}, \mathrm{a})=\left\{\begin{array}{c}
h_{i, a}, i \neq a \\
0, i=a
\end{array}\right.
$$

Recall that our goal is to minimize the power consumption of satellite networks while guaranteeing user QoS during the L handover decision epochs. In our proposed SPO-MDP strategy, it is about choosing a network at each decision epoch so that the expected cost $\mathrm{c}(\mathrm{s}$, a) reaches a minimum. Suppose that the initial state of $M N$ is $s$, let $v(s)$ denotes the minimum expected cost, given as below:

$$
\mathrm{v}(\mathrm{s})=\min _{\pi \epsilon \Pi} v^{\pi}(s)
$$

where the decision state space is $\Pi=\left\{a_{1} \times a_{2} \times \ldots \times a_{L-1} \times a_{L}\right\}$. Then the best handover decision $a^{*}$ at state $\mathrm{s}$ is the solution of the optimal equation:

$$
a^{*}=\arg \min _{a \in A}\left\{c(s, a)+\sum_{s^{\prime} \in S} \theta P\left[s^{\prime} \mid s, a\right] v\left(s^{\prime}\right)\right\}
$$

This equation can be determined by the value iteration algorithm (VIA). Compute $a^{*}$ for each of the state $\mathrm{s}$, we can obtain the best decision policy of SPO-MDP.

\section{Simulation and analysis}

The simulation parameters for both networks are summarized in Table 1.We compare the performance between the proposed SPO-MDP scheme and the MDP scheme in [6]. In this work, performance metrics are the expected power consumption (cost) in satellites, total number of handoffs and call drop rate during the conversation. Fig.4(a) and 4(b)present the expected power consumption(cost) and total number of handoffs with respect to the changes of handover cost. Discount factor of MDP framework is set to be 0.9 and the weight factor is set to be 5.0.From Fig.4(a), it is verified that compared to MDP the expected cost is reduced, that is to say the power consumption of satellite networks is optimized while the user QoS is guaranteed. From Fig.4(a), it is verified that the number of satellite involved handoffs is reduced. And for both strategies the cost decrease slightly and the number of handoff times decreases sharply when the handover cost increases. Fig.4[c] presents the call drop rate of a conversation. It can be seen that the session continuity has been improved by the proposed scheme. 
Table 1 Simulation Parameters

\begin{tabular}{c|l|l|l}
\hline $\begin{array}{l}\text { Notati } \\
\text { on }\end{array}$ & Parameter definition & $\begin{array}{l}\text { Network } \\
\mathrm{m}=1\end{array}$ & $\begin{array}{l}\text { Network } \\
\mathrm{m}=2\end{array}$ \\
\hline$s_{m}$ & Service rate & $0.6 \mathrm{Mbps}$ & $1.2 \mathrm{Mbps}$ \\
\hline $\mathrm{W}$ & weight factor & \multicolumn{2}{|c}{5.0} \\
\hline$H_{r s s}$ & $\begin{array}{l}\text { Highest RSS } \\
\text { requires }\end{array}$ & \multicolumn{2}{|c}{$20 \mathrm{~dB}$} \\
\hline$L_{r s s}$ & $\begin{array}{l}\text { Lowest } \\
\text { requires }\end{array}$ & \multicolumn{2}{|c}{$5 \mathrm{~dB}$} \\
\hline$P_{a, \max }$ & $\begin{array}{l}\text { Maximum } \\
\text { transmission power }\end{array}$ & $6 \mathrm{~W}$ & $12 \mathrm{~W}$ \\
\hline$P_{a, \min }$ & $\begin{array}{l}\text { Minimum } \\
\text { transmission power }\end{array}$ & $2 \mathrm{~W}$ & $4 \mathrm{~W}$ \\
\hline$h_{i, a}$ & $\begin{array}{l}\text { Handover cost from } \\
\text { i to a }\end{array}$ & $8 \mathrm{~W}$ & $8 \mathrm{~W}$ \\
\hline$q_{a}$ & Link quality & \multicolumn{2}{|c}{$q_{a} \in\{0,4,8,12, \infty\} d B$} \\
\hline
\end{tabular}

\section{Summary}

In this paper, we established a power-optimized handover model in Hybrid Satellite-Terrestrial Networks. Further, an improved MDP handover scheme to optimize the satellite power consumption named SPO-MDP was proposed. Simulation results showed that the proposed model and scheme reduced the handoff times involved with the satellite networks, thus giving a better performance in the aspect of satellite power consumption compared to the existing MDP algorithm. In addition, the user satisfaction was enhanced using the strategy brought up in our work.

\section{Acknowledgements}

This work was supported by the National Natural Science Foundation of China (No.61272518) and 2013RC0208 research of the information access technology for complex information system.

\section{References}

[1] Xiaohuan Yan, Y. Ahmet ekerciolu, Sathya Narayanan. A survey of vertical handover decision algorit-hms in Fourth Generation heterogeneous wireless networks [J].Computer Networks.2010 (11).

[2]Sadek, M.; Aissa, S., Personal satellite communication: technologies and challenges, in Wireless Communications, IEEE, vol.19, no.6, pp.28-35, December 2012.

[3] Ahmed, A.; Boulahia, L.M.; Gaiti, D., Enabling Vertical Handover Decisions in Heterogeneous Wire-less Networks: A State-of-the-Art and A Classification, Communications Surveys \& Tutorials, IEEE , vol.16, no.2, pp.776,811, Second Quarter 2014.

[4] Kantubukta, V.; Maheshwari, S.; Mahapatra, S.; Kumar, C.S., Energy and quality of service aware F-UZZY-technique for order preference by similarity to ideal solution based vertical handover decision algorithm for heterogeneous wireless networks, Networks, IET, vol.2, no.3, pp.103,114, Sept. 2013.

[5] Yujae Song; Peng-Yong Kong; Youngnam Han, Power-Optimized Vertical Handover Scheme for Heterogeneous Wireless Networks, Communications Letters, IEEE, vol.18, no.2, pp.277,280, February 2014.

[6] E. Stevens-Navarro, Y. Lin, and V. Wong, An MDP-based vertical handoff decision algorithm for heterogeneous wireless networks, IEEE Trans. Veh. Technol, vol. 57, no. 2, pp. 1243-1254, Mar. 2008.

[7] H.-S. Wang and N. Moayeri, Finite-state Markov channel-a useful model for radio communication channels, IEEE Trans. Veh. Technol., vol. 44, no. 1, pp. 163-171, Feb. 1995. 